\title{
SYMPTOMATIC VENOUS THROMBOEMBOLISM AFTER TOTAL KNEE REPLACEMENT
}

\author{
D. J. WARWICK, S. WHITEHOUSE \\ From the University of Bristol and the Bristol Knee Group, Bristol, England
}

Chemical prophylaxis is known to reduce the venographic prevalence of deep-vein thrombosis (DVT) after total knee replacement (TKR), but it is uncertain whether this affects the incidence of symptoms. Further analysis depends on the basic epidemiology of thromboembolic symptoms. We therefore studied the pattern of such symptoms in a consecutive series of $\mathbf{1 0 0 0}$ patients with primary TKR, with particular reference to risk factors and prophylaxis.

We reviewed all the clinical records and contacted all the patients individually, noting risk factors, prophylaxis, symptomatic pulmonary embolus (PE) or DVT and its timing, death and its causes, and all complications. All the patients wore antiembolism stockings, $83 \%$ had regional anaesthesia and $33.9 \%$ had chemical prophylaxis.

One patient died from PE on the day of surgery, having had no prophylaxis giving a rate of $0.1 \%(95 \%$ CI $0.003 \%$ to $0.56 \%$ ). Symptomatic, radiologically confirmed thromboembolism (VTE) was common with a rate of $10.6 \%(95 \%$ CI $8.7 \%$ to $12.5 \%)$. There was a similar incidence of VTE in those with and without chemical prophylaxis $(10.1 \% v 10.5 \%$, RR 0.96, NS). VTE was more common in patients with risk factors $(15.1 \% v 9.5 \%, \operatorname{RR} 1.59, p=0.02)$ and tended to occur earlier in this group (median day of onset $5 v 7$, $\mathbf{p}=\mathbf{0 . 0 1}$ ). Chemical prophylaxis did not reduce the frequency of symptomatic thromboembolism in either those with risk factors ( $R R \quad 0.81, p=0.5)$ or those without them (RR 0.94, $p=0.8$ ). Haematoma or wound dehiscence was more common in those having chemical prophylaxis $(11.9 \% v 6.9 \%$; RR $1.7395 \%$ CI 1.16 to 2.60). Readmission for symptomatic, radio-logically

D. J. Warwick, MD, FRCS Orth, Lecturer in Orthopaedic Surgery S. Whitehouse, BSc, Grad Stat, Research Assistant

University of Bristol, Avon Orthopaedic Centre, Bristol BS10 5NB, UK.

Correspondence should be sent to Mr D. J. Warwick.

(C)1997 British Editorial Society of Bone and Joint Surgery 0301-620X/97/57761\$2.00 confirmed thromboembolism involved $1.1 \%$ of patients (95\% CI $0.55 \%$ to $2.1 \%$ ). Four patients were readmitted with proven non-fatal $P E$ and six with proven DVT (the latest on day 40).

Our results show that the main risk factor for thromboembolism was TKR itself; chemical prophylaxis did not reduce the incidence of symptomatic thromboembolism but gave an increased perception of side-effects. New prophylactic methods or combinations of methods are needed, with their efficacy compared by randomised controlled studies of both the clinical and the radiological effect.

J Bone Joint Surg [Br] 1997;79-B:780-6.
Received 9 October 1996; Accepted after revision 7 April 1997

The need for thromboprophylaxis after joint replacement is uncertain for three reasons. First, there is good evidence that chemical thromboprophylaxis reduces the venographic prevalence of deep-vein thrombosis (DVT), but there is little to suggest that this results in a reduction of symptomatic incidence. This information is essential for a balanced judgement on prophylaxis, particularly since chemical prophylaxis may carry a risk of side-effects. Secondly, the duration of increased risk is unknown, but is needed to determine the duration of prophylaxis. Thirdly, randomised clinical trials normally include all patients and cannot show whether prophylaxis should be universal or selective (at the surgeon's discretion or for patients believed to have additional risk factors).

To provide some data based on current practice, unaltered by trial conditions, we aimed to describe the usual practice in a large regional orthopaedic centre with 12 consultants performing total knee replacement (TKR), and the resultant pattern of symptomatic thromboembolism. This practice changed during the study period because chemical prophylaxis became more widely used and added the opportunity to discover whether the somewhat random use of prophylaxis was associated with either a reduction of symptomatic thromboembolic events or an increased risk of side-effects. The study could not be designed as strictly prospective or randomised, since this would have altered customary practice and its consequences. We therefore designed a follow-up study of 1000 consecutive primary 


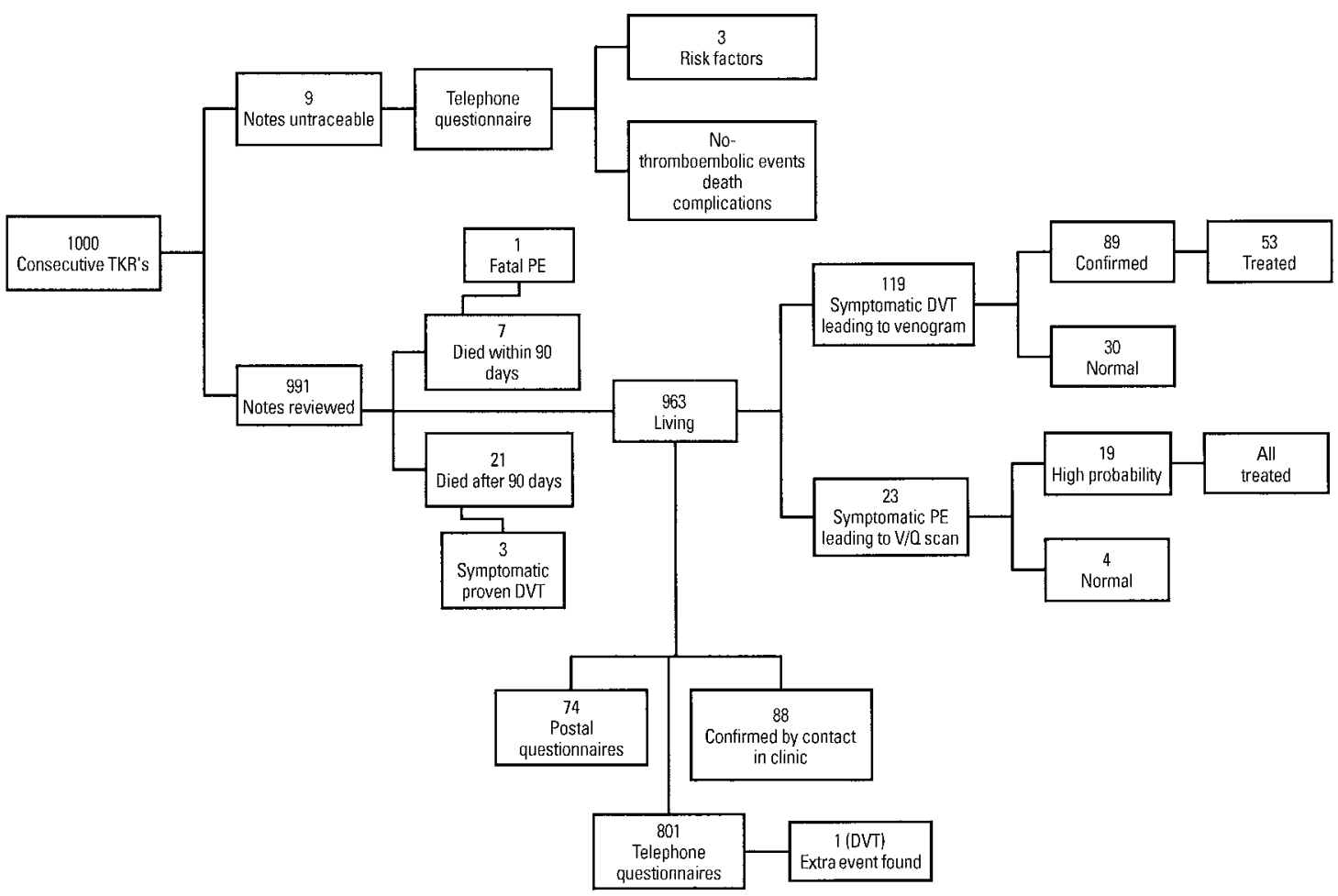

Fig. 1

Process of data acquisition.

TKRs with the aim of acquiring accurate and complete data on the incidence of symptomatic venous thromboembolism during the first three months after surgery.

\section{PATIENTS AND METHODS}

We studied 1000 consecutive patients having a primary TKR as recorded prospectively on a regional database serving 12 consultants, from August 1992 to November 1995. We reviewed in detail the case notes and radiology reports of each patient and confirmed the completeness of the data by postal or telephone enquiries to all patients or by contact with their general practitioners. Death certificates or postmortem reports were obtained for all patients who had died. The specific data recorded are shown in Table I. We recorded all thromboembolic events within 12 weeks as attributable to surgery. ${ }^{1}$ The process of data acquisition is summarised in Figure 1.

Definitions (Table I). The clinical diagnosis by symptoms and signs of DVT or pulmonary embolism (PE) is notoriously unreliable. In cases of reasonable clinical suspicion, patients were investigated by venography or ventilation/ perfusion scanning. Full records of these investigations were obtained.

Symptomatic PE was recorded when a patient had symptoms leading to a ventilation/perfusion scan which showed a high probability of PE.

Symptomatic DVT was noted when a patient had symptoms leading to a venogram which showed a DVT.

Readmission was recorded when a patient, having been discharged from hospital, returned with symptoms leading to a radiologically-confirmed diagnosis of either DVT or PE. Risk factors were recorded as positive when the case notes or a patient interview revealed a previous history of venous

Table I. Data recorded for 1000 patients with TKR

\begin{tabular}{|c|c|c|}
\hline \multirow[t]{4}{*}{ Basic } & Age & \\
\hline & Risk factors & Previous VTE \\
\hline & & Varicose veins \\
\hline & Weight & \\
\hline \multirow[t]{5}{*}{ Prophylaxis } & Stockings & \\
\hline & Spinal/epidural & \\
\hline & Heparin & \\
\hline & Warfarin & \\
\hline & LMWH & \\
\hline \multirow[t]{3}{*}{ Prophylaxis } & Dose & \\
\hline & Start point & \\
\hline & Finish point & \\
\hline \multirow[t]{7}{*}{ Outcome } & Symptomatic DVT & Description of venogram \\
\hline & Symptomatic PE & V/Q scan (high probability) \\
\hline & Death & Postmortem or death certificate \\
\hline & Time of onset & In hospital \\
\hline & & Readmission \\
\hline & Readmission & Diagnosis \\
\hline & & Time after surgery \\
\hline \multirow[t]{3}{*}{ Complications } & Haematoma & Recorded in notes \\
\hline & Wound dehiscence & \\
\hline & GI bleed, CVA, MI & \\
\hline
\end{tabular}




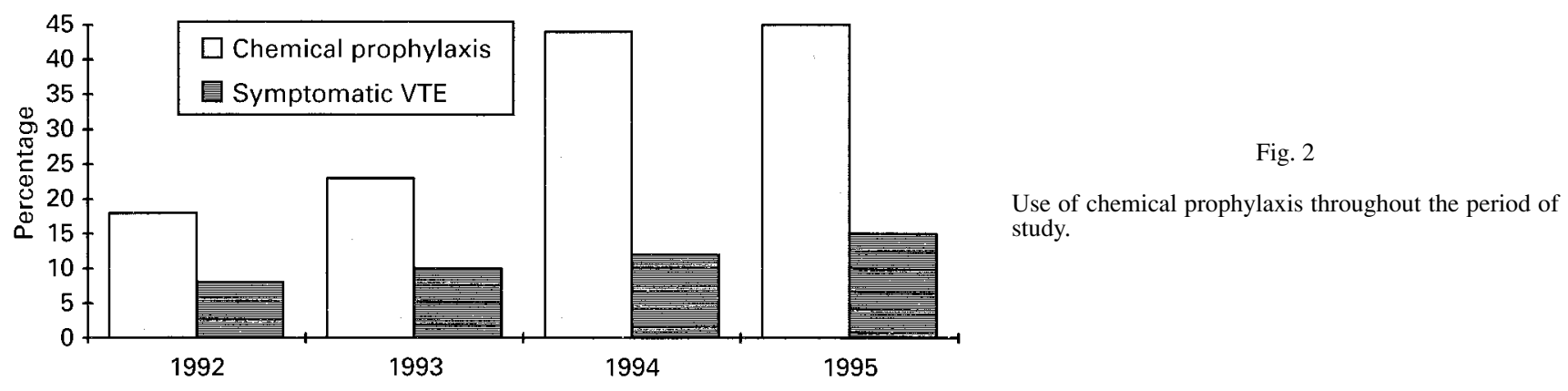

Table II. Deaths within 90 days of TKR

\begin{tabular}{cll}
\hline Day & Cause & Verification \\
\hline 0 & Pulmonary embolism & Postmortem \\
2 & Cerebrovascular accident & Postmortem \\
3 & Renal failure & Death certificate \\
11 & Myocardial infarct & Postmortem \\
23 & Peritonitis & Death certificate \\
38 & Bronchopneumonia & Death certificate \\
86 & Bronchopneumonia & Death certificate \\
\hline
\end{tabular}

thromboembolism or of varicose veins.

We recorded full details of chemical prophylaxis including the time of starting, the duration, and the dosage. The use of graduated compression stockings and of regional anaesthesia, both of which are regarded as adjunctive prophylactic measures, was determined from the nursing and anaesthetic charts.

Statistics. Data were recorded on a purpose-modified database (Microsoft Access), and the incidence rates and relative risks were calculated with $95 \%$ confidence intervals (CI); (Confidence Interval Analysis, BMJ, London). Categorical variables were compared using the chi-squared test. Ordinal variables were compared with the Mann-Whitney $\mathrm{U}$ test, and continuous variables with the $t$-test.

\section{RESULTS}

The records for nine of the 1000 patients could not be located and therefore data on prophylaxis were not available for them. Direct contact with these patients enabled us to assess the presence of risk factors and the occurrence of clinical DVT or PE. Routine direct contact with patients by letter or telephone detected only one additional complication which would not have been discovered.

Types of prophylaxis. All patients used graduated compression stockings, and $83.3 \%$ had regional anaesthesia. A total of 336 patients $(33.9 \%)$ had chemical prophylaxis; this was by low-molecular-weight heparin (LMWH) in 75\%, unfractionated heparin in $17.2 \%$, warfarin in $3.9 \%$, and warfarin with heparin in $3.9 \%$. Chemical prophylaxis was increasingly used during the study period (Fig. 2), and the proportion of patients having LMWH rose from $25 \%$ to
$95 \%$ of all chemical prophylaxis during this period. The proportion of those developing symptomatic venous thromboembolism (VTE) was similar in those receiving prophylaxis $(37$ of $366,10.1 \%$ ) and in those without chemical prophylaxis $(69$ of $655,10.5 \%)$. The relative risk was $0.96(95 \%$ CI $0.66 \%$ to $1.40 \%$; chi-squared test $=0.04$, $\mathrm{p}=0.84)$. The particular choice of prophylaxis was not governed by a protocol or guidelines, but was made empirically according to the surgeon's beliefs and risk assessment.

Death. Seven patients died within 90 days of knee replacement $(0.7 \%, 95 \%$ CI $0.2 \%$ to $1.4 \%)$ (Table II). The only patient who died of proven PE had no risk factors other than obesity and had been given no chemical prophylaxis. The two deaths reported to be due to bronchopneumonia could be attributed to PE, although the clinical picture suggested that this was improbable. The fatal PE rate was therefore 1 of $1000(0.1 \%, 95 \%$ CI $0.003 \%$ to $0.56 \%)$.

Symptomatic PE (Fig. 3). Ventilation/perfusion scanning was performed in 23 patients for symptoms consistent with PE. Nineteen of these were positive $(1.9 \%$ of the study population, $95 \%$ CI $1.2 \%$ to $3.0 \%$ ). Two of those with proven PE also had venographically-proven symptomatic DVT (one major calf, one popliteal). Both of these patients had been given chemical prophylaxis. Three additional patients who were having treatment for a proven major DVT subsequently had symptoms consistent with PE, but this was not confirmed radiologically.

Symptomatic DVT (Fig. 3). Venography was performed in 119 patients for symptoms consistent with DVT. This confirmed the clinical diagnosis in 89 patients $(8.9 \%$ of study population, $95 \%$ CI $7.2 \%$ to $10.8 \%$ ). There were 5 thigh, 8 popliteal, 2 major calf with small femoral, 50 major calf and 24 minor calf thrombi. Of the 106 with positive results 72 were judged to have a DVT or PE which required treatment with full therapeutic anticoagulation.

Duration of risk (Fig. 3). Day 6 was the median day of onset of PE and DVT. The patients with risk factors developed clinical symptoms earlier than those without such factors (median day of onset day 5 against day 7; U statistic $812.5, \mathrm{p}=0.01$ ). The median day of onset of groups with or without chemical prophylaxis was also day 6 .

Ten patients (10 of $893,1.1 \%, 95 \%$ CI $0.5 \%$ to $2.1 \%$ ) were readmitted with thromboembolism. Four had proven 


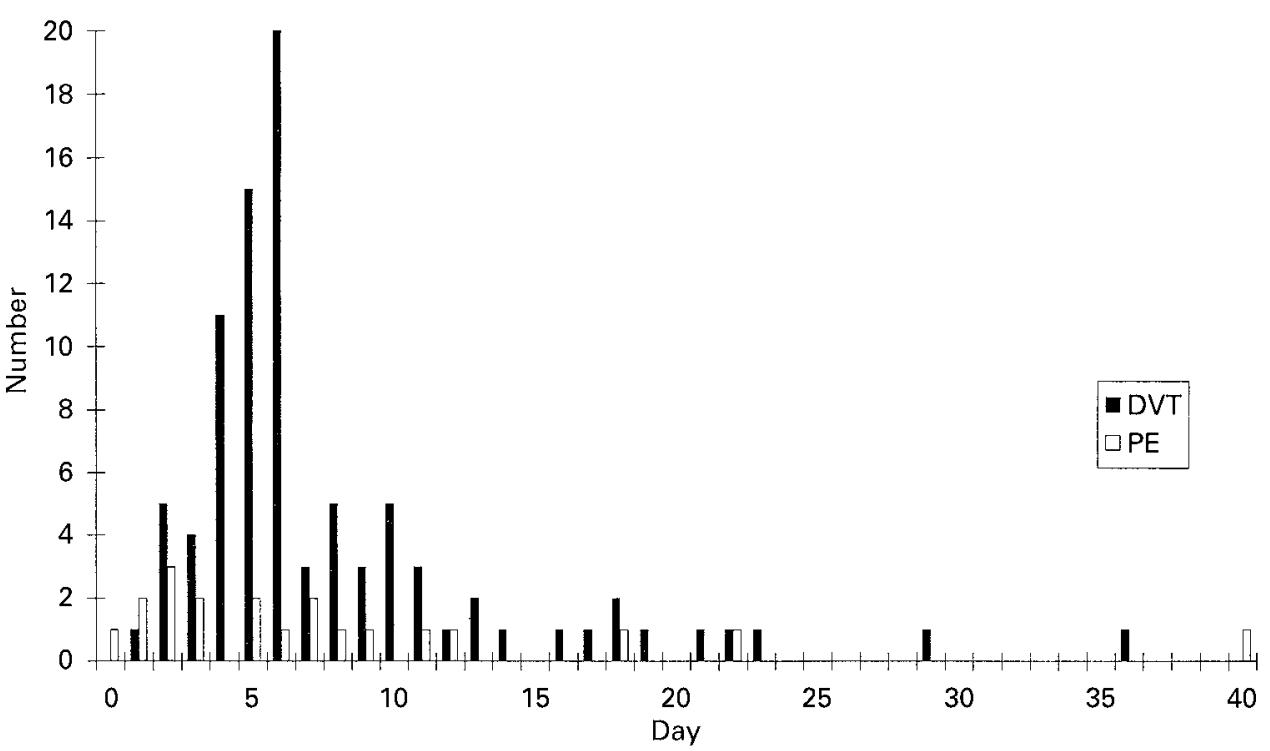

Fig. 3

Time of clinical presentation of symptomatic, radiographically-proven PE and DVT.

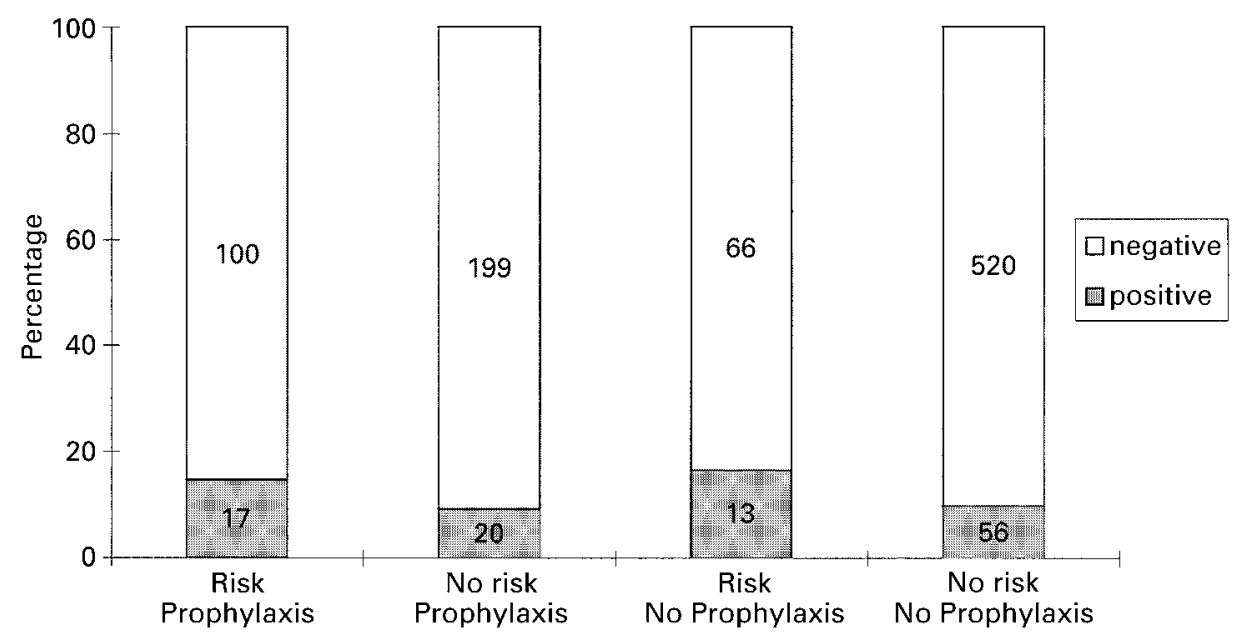

Fig. 4

The effect of risk factors and prophylaxis on the event rate.

non-fatal PE (days 12, 18, 22 and 40). None of them had risk factors, and one had had chemical prophylaxis at the original admission. Six patients with DVT (days 10, 19, 22, 23, 29 and 36) were readmitted: none had risk factors, four had in-hospital prophylaxis. There was one major calf DVT and five minor calf DVTs.

Complications. The proportion of patients developing a haematoma or wound dehiscence was 40 of 336 (11.9\%) with chemical prophylaxis and 45 of 655 (6.9\%) without (relative risk with prophylaxis $1.73,95 \%$ CI 1.16 to 2.60 chi-squared test $=7.58, \mathrm{p}=0.006$ ).

Risk factors (Fig. 4). Of the total, 199 (19.9\%) had risk factors (previous VTE in 86, varicosed veins in 101, both in 12): 76 of $801(9.5 \%)$ of those without apparent risk factors and 30 of $199(15.1 \%)$ of those with, developed sympto- matic VTE (relative risk 1.59 , 95\% CI 1.07 to 2.35 , chisquared test $=0.02$ ). The mean age of those with VTE was 71.8 years (SD 6.98) and of those without 71.3 (SD 9.7) $(t$ test, $\mathrm{p}=0.62)$. The mean weight of those with VTE was $77.9 \mathrm{~kg}$ (SD 14.5) and of those without 74.9 (SD 14.9) (twotailed $t$-test, $\mathrm{p}=0.05$ ). The selective use of chemical prophylaxis did not alter the frequency of symptomatic VTE in those with risk factors (RR $0.81,95 \%$ CI 0.42 to 1.57 , chisquared test $=0.39, \mathrm{p}=0.50)$ or those without $(\mathrm{RR} 0.94$, $95 \%$ CI 0.58 to 1.53 , chi-squared test $=0.06, p=0.80$ ).

\section{DISCUSSION}

We obtained our data from a consecutive series of 1000 patients having TKR. The hospital records were validated 
as a data source since only one extra event was detected by further contacts with the patient or general practitioner. The study was designed to discover the profile of symptomatic thromboembolism associated with existing practice. Such a profile cannot be acquired from a randomised study since the necessary methodology, such as the inclusion of all patients regardless of risk factors and the use of routine venography, would obscure the information which we aimed to analyse.

Previous studies. Little is known about the incidence and timing of clinical venous thromboembolism after TKR. In two series in which chemical prophylaxis had not been used, Khaw et $\mathrm{al}^{2}$ reported one fatal PE in 499 patients $(0.2 \%, 95 \%$ CI $0.005 \%$ to $1.0 \%)$ and $\mathrm{Kim}^{3}$ reported none in $244(0 \%, 95 \%$ CI $0 \%$ to $1.5 \%)$. These figures are consistent with our rate of $0.1 \%$. Mohr et $\mathrm{al}^{4}$ found clinical thromboembolism in four of 152 patients with TKR not having prophylaxis $(2.6 \%, 95 \% \mathrm{CI} 0.7 \%$ to $6.6 \%)$, less than the frequency of $8.7 \%$ to $12.5 \%$ in our series. Other studies have indicated a symptomatic PE rate of $0 \%$ to $2.1 \%$ regardless of prophylaxis; we could find no further data on the rate of symptomatic DVT. ${ }^{2,3,5-7}$

Importance of symptomatic venous thromboembolism. Patients with proven PE (high probability V/Q scans) and those with major calf and proximal DVTs are treated to reduce the chance of recurrence. Treatment by full anticoagulation after a joint replacement carries a complication rate of up to $45 \%$, which adds to the potential cost and morbidity of thromboembolism. One patient in our series required a local flap and eventually an arthrodesis after developing a wound haematoma during therapeutic anticoagulation. Symptomatic DVT is reported to lead to clinical chronic venous insufficiency in $30 \%$ to $90 \%$ of patients within five years, ${ }^{9}$ but it is not clear that therapeutic anticoagulation reduces this risk.

Symptomatic VTE, sustained by $10.6 \%$ in our series, causes morbidity in addition to the costs of investigation, drugs, monitoring, accommodation and the treatment of complications of therapeutic anticoagulation. Clinically effective prophylaxis is an urgent requirement, but did not seem to be provided by the practice in our study.

Importance of small calf thrombi. Leg swelling and calf pain are common after TKR, as are calf thromboses (some $60 \%$ ). For this reason some patients with symptoms consistent with a DVT may have had a venogram which revealed a coincidental, but not causal, thrombosis, regardless of whether that thrombosis was the cause of the symptoms. The poor specificity of the clinical diagnosis of DVT $^{10}$ has been emphasised by our finding that, of 119 venograms for symptoms consistent with DVT, 30 were normal $(25 \%)$.

The clinical significance of calf DVT is a controversial subject. Some authors report that treatment does not reduce symptoms, or the risk of propagation. ${ }^{11,12}$ Despite this it is known that a calf DVT after knee replacement can propagate into the proximal veins, ${ }^{13,14}$ leading to a risk of $\mathrm{PE}{ }^{7}$
Serial imaging of calf thrombi has been recommended to detect and allow treatment of propagating thrombi. We have used an empirical, but pragmatic, approach; we treat major calf and proximal thrombi, but ignore small calf thrombi.

Venographic evidence in thromboprophylaxis after knee replacement. Randomised studies have shown that LMWH is superior to warfarin and to standard heparin in reducing the venographic prevalence of DVT, ${ }^{15-22}$ and there is very little evidence to support the use of aspirin or dextran. Even with LMWH prophylaxis there is only a $50 \%$ reduction in venographic DVT, from a control prevalence of $60 \%$ to $30 \%$.

Mechanical prophylaxis is attractive, because there is no risk of haemorrhagic side-effects. There is some evidence that intermittent pneumatic calf compression reduces venographic DVT, ${ }^{23-25}$ and foot impulse pumps, which are less intrusive, have been shown to reduce effectively the venographic prevalence of DVT, particularly of proximal thrombi. $^{26,27}$ As yet there are no valid reports comparing mechanical methods with the use of LMWH.

Value of existing trial data. Definitive comment on the efficacy and safety of thromboprophylaxis should be made only on data from adequately-sized randomised studies which use specific protocols and objective outcomes; our study was not designed to fulfil these criteria. The usual randomised trials use venography as an outcome. This indicates prevalence, the frequency at one moment in time, but gives no information on incidence, the overall number of events. In addition, venography is an unvalidated substitute for the real outcome of interest, which must be symptomatic VTE.

Our study has raised the possibility that current prophylactic protocols, all based on venographic evidence, may not alter the symptomatic expression of thromboembolism. This requires further study, particularly when an additional risk is associated with prophylaxis. Future trials should emphasise the clinical rather than the radiological outcomes.

The effect of bias in our study design. The discrepancy which we found between the risk reduction expected from venographic trial data, and the disappointingly unaltered rate of symptomatic event needs consideration. One possibility is that the expected gain suggested by venographic studies was not translated into clinical benefit. A second possibility is that compliance may not have been optimal, although review of the prescription charts showed that medication was given correctly in nearly all cases.

There were two other potential sources for bias. Complications may have been sought, investigated and recorded more thoroughly in the prophylaxis group, perhaps because of cynicism concerning chemical agents. The second possible bias, increasing awareness of VTE, was that the wider prescribing of thromboprophylaxis led to a lower threshold for radiological investigation of symptoms. Our results suggest that neither of these potential biases was important. 
The proportion of those having venograms or V/Q scans was similar in patients having chemical prophylaxis (53 of $336,15.8 \%)$ and in those without (85 of $655,13.0 \%)$ (chisquared test $=1.45, \mathrm{p}=0.2$ ). Similarly, the proportion of positive venograms and positive V/Q scans was also similar in the prophylaxis and no-prophylaxis groups (37 of 53, $70 \%$ and 68 of $85,80 \%$ respectively, chi-squared test $=1.86, \mathrm{p}=0.17)$.

Safety of chemical prophylaxis after knee replacement. We found a higher relative risk of haemorrhagic complications in the prophylaxis than in the no-prophylaxis group (relative risk 1.73). Our study design does not exclude the possible bias that bleeding complications were more likely to be recorded after chemical prophylaxis because of preconceptions of this risk. Few data are available to confirm or refute the concerns of some surgeons ${ }^{28}$ that chemical prophylaxis is associated with bleeding. One small randomised controlled study has compared LMWH with placebo after TKR; ${ }^{22}$ this showed no apparent difference in major haemorrhagic complications.

Risk factors. We found the incidence of VTE to be higher in those with risk factors (RR 1.59), but even then $9.5 \%$ of patients with no obvious clinical risk factors developed symptomatic thromboembolism, which means that selective prophylaxis is inappropriate. The aim should be universal prophylaxis by a clinically-effective, cost-effective and safe method. More research is essential before the most appropriate method can be recommended.

Prolonged prophylaxis. Our finding of a $1.1 \%$ incidence of problems after discharge raises the question of prolonged prophylaxis after leaving hospital. Five of the six clinically evident DVTs occurring after discharge were small and of dubious significance. This suggests that the risk of thromboembolism after discharge is too low to justify an extension of prophylaxis after knee replacement. There are no trial data to support such a policy, and any such investigations must carefully consider cost, efficacy, safety and compliance.

Conclusions. We have tried to establish a profile of symptomatic thromboembolism in mixed clinical practice to provide a background for discussion of thromboprophylaxis. Symptomatic, radiologically-confirmed thromboembolism is common, usually within ten days of surgery, and significant events are unusual after discharge.

Fatal PE is rare, and the main risk factor is knee replacement itself, not any clinically-identifiable factor. Chemical prophylaxis may not influence the clinical incidence of thromboembolic events; such prophylaxis may carry an increased risk of wound complications.

Further studies are required to find the most effective, safe and economic form of prophylaxis, with consideration of clinical as well as venographic outcome.

We wish to thank the Wishbone Trust for funding this study and the Bristol Knee Group for allowing us to work with their database and study their patients.

No benefits in any form have been received or will be received from a commercial party related directly or indirectly to the subject of this article.

\section{REFERENCES}

1. Seagroatt V, Tan HS, Goldacre M, et al. Elective total hip replacement: incidence, emergency readmission rate and postoperative mortality. Br Med J 1991;303:1431-5.

2. Khaw FM, Moran CG, Pinder IM, Smith SR. The incidence of fatal pulmonary embolism after knee replacement with no prophylactic anticoagulation. J Bone Joint Surg [Br] 1993;75-B:940-1.

3. Kim Y-H. The incidence of deep vein thrombosis after cementless and cemented knee replacements. J Bone Joint Surg [Br] 1990;72-B:77983.

4. Mohr DN, Silverstein MD, Ilstrup DM, Heit JA, Morrey BF. Venous thromboembolism associated with hip and knee arthroplasty: current prophylactic practices and outcomes. Mayo Clin Proc 1992; 67:861-70.

5. Stulberg BN, Insall JN, Williams GW, Ghelman B. Deep-vein thrombosis following total knee replacement: an analysis of six hundred and thirty-eight arthroplasties. J Bone Joint Surg [Am] 1984; 66-A:194-201

6. Lotke PA, Ecker ML, Alavi A, Berkowitz H. Indications for the treatment of deep venous thrombosis following total knee replacement. J Bone Joint Surg [Am] 1984;66-A:202-8.

7. Haas SB, Tribus CB, Inall JN, Becker MW, Windsor RE. The significance of calf thrombi after total knee arthroplasty. J Bone Joint Surg [Br] 1992;74-B:799-802.

8. Patterson BM, Marchand R, Ranawat C. Complications of heparin therapy after total joint arthroplasty. J Bone Joint Surg [Am] 1989; 71-A:1130-4.

9. Philbrick JT, Becker DM. Calf deep venous thrombosis: a wolf in sheep's clothing? Arch Intern Med 1988;148:2131-8.

10. Wells PS, Hirsh J, Anderson DR, et al. Accuracy of clinical assessment of deep vein thrombosis. Lancet 1995;345:1326-30.

11. Strandness DE. Editorial. Thrombus propagation and level of anticoagulation. J Vasc Surg 1990;12:497-8.

12. Solis MM, Ranval TJ, Nix ML, et al. Is anticoagulation indicated for asymptomatic postoperative calf vein thrombosis? J Vasc Surg 1992; 16:414-9.

13. Maynard MJ, Sculco TP, Ghelman B. Progression and regression of deep vein thrombosis after total knee arthroplasty. Clin Orthop 1991; 273:125-30.

14. Oishi CS, Grady-Benson JC, Otis SM, Colwell CW Jr, Walker RH. The clinical course of distal deep venous thrombosis after total hip and knee arthroplasty, as determined with duplex ultrasonography. J Bone Joint Surg [Am] 1994;76-A:1658-63.

15. Hull R, Raskob G, Pineo G, et al. A comparison of subcutaneous low-molecular weight heparin with warfarin sodium for prophylaxis against deep-vein thrombosis after hip or knee implantation. $N$ Engl J Med 1993;329:1370-6.

16. Leclerc JR, Geerts WH, Desjardins BI, et al. Prevention of venous thromboembolism after knee arthroplasty: a randomised, double blind trial comparing a low molecular weight heparin fragment (enoxaparin) to warfarin. Ann Int Med 1996;124:619-26.

17. Spiro TE, Fitzgerald RH, Trowbridge AA, et al. Enoxaparin a low molecular weight heparin and warfarin for the prevention of venous thromboembolic disease after elective knee replacement surgery. Thromb Haem 1995;73:978.

18. RD Heparin Arthroplasty Group. RD heparin compared with warfarin for prevention of venous thromboembolic disease following total hip or knee arthroplasty. J Bone Joint Surg [Am] 1994;76-A:117485 .

19. Heit J, Berkowitz S, Bona R, et al. Efficacy and safety of Normiflo (a LMWH) compared to warfarin for the prevention of venous thromboembolism following total knee replacement. Thromb Haem 1995;73:978. 
20. Colwell CW, Spiro TE, Trowbridge AA, et al. Efficacy and safety of enoxaparin versus unfractionated heparin for prevention of deep vein thrombosis after elective knee arthroplasty. Clin Orthop 1995; 321:19.

21. Fauno P, Suomalainen AO, Rehnberg V, et al. Prophylaxis for the prevention of venous thromboembolism after total knee arthroplasty: a comparison between unfractionated and low-molecular-weight heparin. J Bone Joint Surg [Am] 1994;76-A:1814-8.

22. Leclerc JR, Geerts WH, Desjardins L, et al. Prevention of deep vein thrombosis after major knee surgery: a randomised, double-blind trial comparing a low molecular weight heparin fragment (enoxaparin) to placebo. Thromb Haem 1992;67:417-23.

23. Hull R, Delmore TJ, Hirsh J, et al. Effectiveness of intermittent pulsatile elastic stockings for the prevention of calf and thigh vein thrombosis in patients undergoing elective knee surgery. Thromb Res $1979 ; 16: 37-45$.
24. McKenna R, Galante J, Bachmann F, et al. Prevention of venous thromboembolism after total knee replacement by high-dose aspirin or intermittent calf and thigh compression. Br Med J 1980:514-7.

25. Haas SB, Insall JN, Scuderi GR, Windsor RE, Ghelman B. Pneumatic sequential-compression boots compared with aspirin prophylaxis of deep-vein thrombosis after total knee arthroplasty. $J$ Bone Joint Surg [Am] 1990;72-A:27-31.

26. Westrich GH, Sculco TP. Prophylaxis against deep venous thrombosis after total knee arthroplasty: pneumatic plantar compression and aspirin compared with aspirin alone. J Bone Joint Surg [Am] 1996; 78-A:826-34.

27. Wilson NV, Das SK, Kakkar VV, et al. Thromboembolic prophylaxis in total knee replacement: evaluation of the A-V Impulse system. J Bone Joint Surg [Br] 1992;74-B:50-2.

28. Murray DW, Carr AJ, Bulstrode CJK. Pharmacological prophylaxis and total hip replacement. J Bone Joint Surg [Br] 1995;77-B:3-5. 